\title{
Effect of one bout of local vibration exercise with blood flow restriction on neuromuscular and hormonal responses
}

\author{
W-C Chen ${ }^{1,2}, \mathrm{C}-\mathrm{M} \mathrm{Wu}{ }^{3}, \mathrm{Z}-\mathrm{Y} \mathrm{Cai}{ }^{4}$ \\ ${ }^{1}$ Center for General Education, Chang Gung University of Science and Technology, Taoyuan, Taiwan \\ ${ }^{2}$ Department of Otolaryngology-Head and Neck Surgery, Linkou Chang Gung Memorial Hospital, Taoyuan, Taiwan \\ ${ }^{3}$ Department of Leisure and Sports Management, Cheng Shiu University, Kaohsiung City, Taiwan \\ ${ }^{4}$ Division of Physical and Health Education, Center for General Education, National Sun Yat-sen University, \\ Kaohsiung City, Taiwan
}

Received: June 20, 2017

Accepted: March 29, 2018

\begin{abstract}
This study aimed to investigate the effects of single local vibration (LV) with and without blood flow restriction (BFR) on muscle activity and hormonal responses. A total of 12 physically inactive males were exposed to 10 sets of intermittent LV $(35-40 \mathrm{~Hz})$ on unilateral mid-quadriceps in the supine lying position and LV + BFR (inflated to $140 \mathrm{mmHg}$ ) sessions in a repeated-measures randomized crossover design, with a 1-week interval separating the sessions. The results indicated that the electromyography values from the rectus femoris during LV + BFR were greater than those during LV $(p<0.05)$. LV + BFR caused a minor increase in the lactate (LA) response $(p<0.05)$; LV with or without BFR failed to elicit change in growth hormone (GH) and testosterone (T) levels $(p>0.05)$. Cortisol (C) levels were decreased postexercise in both the sessions $(p<0.05)$. In conclusion, BFR elicited higher increase in muscle activity and metabolic response, but it did not induce hormonal responses. The exposure of LV and LV + BFR may only have a relief effect as detected by the reduction in C levels, probably because the LV did not elicit sufficient stimulus to the muscles.
\end{abstract}

Keywords: electromyography, lactate, growth hormone, testosterone, cortisol

\section{Introduction}

In recent decades, research has indicated that $20 \%-30 \%$ of one-repetition maximal of lowintensity resistance training combined with blood flow restriction (BFR) results in muscle hypertrophy and strength gain in adaptations similar to those produced by high-intensity resistance training $(23,33)$. This emerging strength training method, where the proximal portion of exercising muscles is typically compressed by wrapping an inflated cuff or wrap, is termed occlusion training or BFR resistance exercise (33). BFR resistance training allows individuals to train their muscles at a desired intensity while keeping the load relatively low, which benefits those who are unable to tolerate resistance training at heavy load $(23,33)$. Based on this rationale, BFR resistance training seems to be both an effective and safe alternative to heavy resistance training.

\footnotetext{
Corresponding author: Zong-Yan Cai

Division of Physical and Health Education, Center for General Education, National Sun Yat-sen University

No. 70, Lianhai Rd., Gushan District, Kaohsiung City 804, Taiwan

Phone: +886 75252 000, ext. 5872; Fax: +886 35255 851; E-mail: flameyan@yahoo.com.tw
} 
BFR resistance training has primarily been reported to have an additive effect on strength training adaptations (e.g., muscle hypertrophy and strength gain) when compared with low-load resistance exercise without $\operatorname{BFR}(22,33)$. Training adaptation may be viewed as the cumulative effects of multiple acute exercise responses. With regard to acute effects of resistance exercise, one bout of conventional high-intensity resistance exercise is often accompanied by increased metabolic and hormonal responses $(7,39)$, and so is BFR resistance exercise $(31,34,40)$. During BFR resistance exercise, venous return of blood flow from muscles is occluded, which may cause a hypoxic environment (36) and has increased metabolic stress [e.g., phosphocreatine depletion, diprotonated phosphate elevation, intramuscular $\mathrm{pH}$ reduction, and lactate (LA) accumulation] when compared with lowload resistance exercise alone $(33,35)$. Evidence suggests that metabolic stress appears to play the dominant role in mediating the potent hypertrophic effects with BFR resistance training (32). Studies have reported that the LA level, which is a component of the metabolic stress response, is exaggerated in the BFR resistance exercise-associated hypoxic environment (36). In addition, increased LA levels following BFR exercise were correlated with increased muscle activation (40) and were considered to potentially play a part in stimulating muscle activation (40). In addition, participants displayed a higher rating of perceived exertion (RPE) response to resist force when undergoing BFR resistance exercise (40). Increased LA levels would partially evoke subsequent anabolic factor release $(22,24,32)$. Accumulating evidence has shown that BFR resistance exercise elicits an additive effect on LA $(34,40)$ and both hormonal $(31,34)$ and muscle activation responses $(40,41)$ compared with low-intensity resistance exercise without BFR. Altogether, LA accumulation derived from BFR may play an important role in eliciting a greater hypertrophic stimulus.

Both resistance exercise and BFR resistance exercise, when performed appropriately, will effectively increase muscle strength and mass $(13,15,22,23,33)$, and the importance of performing regular resistance exercise has been emphasized by sports-related associations (15). However, for some populations, engaging in resistance exercise can be difficult. Hence, strategies that involuntarily invoke muscle contraction for an alternative training modality are important. Given that the additive effect of BFR resistance exercise is often observed under low-intensity resistance exercise, there is a spurring speculation that even passive muscle contractions can lead to intensities comparable with those during low-intensity resistance exercise and can induce an additive exercise effect when combined with BFR.

Vibration training has commonly been used as an alternative resistance training modality (6). Vibration can contract the muscles involuntarily. This muscle contraction process is termed tonic vibration reflex (TVR), during which muscle spindles work through the stretch reflex, thereby facilitating the activation of Ia-motoneurons, leading to involuntary muscle contraction (19). Two primary forms of vibration are relevant to training, wholebody vibration (WBV), and local vibration (LV). WBV, also known as indirect vibration, occurs when a vibration platform repeatedly and rapidly lifts an individual up and down and transmits the vibrations to the targeted muscles. In contrast, LV, also known as direct vibration, involves only the specific muscle that is vibrated by placing a vibrator on it. Extensive evidence has shown that WBV training-induced muscle adaptation is similar to that observed with resistance exercise, e.g., it can both improve the strength $(6,10)$ and promote an increase in muscle mass (26). In addition, acute WBV + BFR can induce the activation and subsequent differentiation of satellite cells (2), suggesting that WBV + BFR might represent a viable anabolic stimulus. Although numerous aspects of WBV have already been investigated, LV exposure has received less attention. A possible mechanism of eliciting 
involuntary muscle contraction is similar between LV and WBV since both augment muscle spindle activity, which elicits a stretch reflex response. However, LV does not include changes in the gravitational load that are integral to WBV. Exposure to LV might be as safe as WBV because the stress given to the head can be minimized during vibration, and it is suitable for people who cannot tolerate standing on a WBV platform. Moreover, the small portable vibrator for LV supports its use as a training modality. Although studies on the physiological effects of acute or cumulative LV exposure are still in their infancy, previous studies on resistance exercise with superimposed LV had reported an additive effect on both muscle activity and LA levels when LV was applied to resistance exercise compared with resistance exercise alone $(3,8)$. An isolated LV exposure study also found that muscle activity was enhanced immediately following LV (29). Furthermore, acute LV exposure significantly influences the levels of certain hormones (18). Moreover, leg extension strength increased after regular LV training $(18,37)$. These studies suggest that LV may cause a similar response to that with resistance training. However, whether acute LV + BFR would have an additive response compared with LV alone is currently not clear.

Considering the aforementioned research, the purpose of this study was to investigate the acute effect of LV + BFR on muscle activation and metabolic and hormonal responses in untrained populations and to investigate whether LV + BFR would produce an additive response compared to that with $\mathrm{LV}$ alone. In addition, physiological parameter such as RPE was evaluated in this study.

\section{Materials and Methods}

\section{Subjects}

A total of 12 healthy males (age: $20.67 \pm 0.14$ years, height: $172.75 \pm 1.95 \mathrm{~cm}$, and body mass: $65.08 \pm 2.21 \mathrm{~kg}$ ) participated in this study. The sample size was based on the LA parameter, because studies have indicated that increased metabolic acidosis in the form of LA accumulation is partly involved in growth hormone $(\mathrm{GH})$ and testosterone $(\mathrm{T})$ secretion $(22,24,32)$ and may influence cortisol (C) levels (17). In addition, LA may play a part in stimulating the muscle activation. Using $\mathrm{G}^{*}$ Power 3.1, an a priori sample size estimation based on LA showed that 10 participants were needed for a potential difference between sessions with a moderate effect size of 0.4 , an alpha level of 0.05 , and a power level of 0.8 . All the participants were determined to be physically inactive through screening with the International Physical Activity Questionnaire - Short Form (16). No participants reported that they were smokers or alcohol and coffee drinkers. The study was approved by the Institutional Review Board of Kaohsiung Medical University Chung-Ho Memorial Hospital, Kaohsiung, Taiwan and was conducted in accordance with the ethical standards of the Declaration of Helsinki protocol, and written informed consent was obtained from all subjects.

\section{Study design}

A week before the experiment, the participants were familiarized with the experimental procedures and devices. Subsequently, they completed two treatment sessions, i.e., LV + BFR (intervention: LV + BFR) and LV (intervention: LV alone), using a repeated-measures randomized crossover design with the sessions separated by a 1-week interval. The participants in the LV session applied four vibrators locally in the front of unilateral midthigh with frequencies between 35 and $40 \mathrm{~Hz}$ and acceleration between 1.96 and $2.57 \mathrm{~g}$. 
The participants in the LV + BFR session received the same LV treatment, but an external pressure of $140 \mathrm{mmHg}$ was applied using inflatable cuffs over the proximal portion of the thigh muscles. To realize the participants' RPE and muscle activity during each session, RPE using the Borg CR-10 Scale and thigh electromyography (EMG) activity measurements were conducted to document and compare any significant changes between the treatment sessions. In addition, fasting blood samples before and after treatment were collected to assess LA levels and hormonal responses. For the experiments, the participants were instructed to refrain from consuming alcohol, caffeine, or nutritional supplements for $24 \mathrm{~h}$ before the treatment sessions and to avoid strenuous exercise for $48 \mathrm{~h}$ before the sessions. To minimize diurnal variation, all measurements were obtained at the same time in the morning of the experiment day (between 9:00 and 11:00 a.m.).

\section{Procedure}

The study was performed during the summer and lasted for 3 weeks. On each assessment day, participants arrived at the laboratory and rested for 10-15 min. A registered nurse then collected fasting blood samples from participants who were in the sitting position as a baseline measurement. Participants then performed a standardized warm-up, consisting of 5 min of bicycling on a cycle ergometer (pedal cadence was set at $50 \mathrm{rpm}$ against no resistance), followed by $3 \mathrm{~min}$ of static stretching for the quadriceps and hamstrings. After a warm-up, $\mathrm{Ag} / \mathrm{Agcl}$ circular bipolar surface electrodes (2-cm interelectrode distance) were attached to the belly of the rectus femoris (RF) muscle of the participant's non-dominant leg to collect EMG signals during each exercise session. On the anterior thigh, the RF is selected for analysis because it was the muscle that was primarily being vibration-treated. The non-dominant leg was defined as the individual's self-reported poor performance of one side of the body compared with that of the other side as the last foot before a jumper takes off. The nondominant leg was treated to emphasize the character of physically inactive populations, in case an individual may express a preference for using a particular limb (dominance) for performing daily activities. To begin each experiment, a maximal voluntary isometric contraction (MVC) of leg extension was determined with the knee angle at $70^{\circ}$ and the hip angle at $110^{\circ}$ (3-s duration) to induce a maximal EMG response for normalization of the peak EMG signals recorded during the $\mathrm{LV}+\mathrm{BFR}$ and $\mathrm{LV}$ sessions. Verbal encouragement was given during MVC. The participants then completed the given LV treatment with or without BFR in the supine lying position with legs straight. Four vibrators were centered on the mid part of the quadriceps using bandages below the electrodes. During application of these sessions, the participants were told that it is not required to maintain isometric contraction of the quadriceps. At the end of each set, the participants were asked to rate their perceived exertion. Immediately, posttreatment as well as 15- and 30-min posttreatment blood samples were collected again from each participant in the sitting position for subsequent analysis.

\section{LV devices and protocol applied}

The custom-made vibration devices and intensity were adapted from Tankisheva et al. (37), which have previously been reported to increase participants' strength when used regularly. Briefly, the device consisted of one control unit, four vibrators connected to the control unit, and a medically approved power supplier. Each vibrator had an encoder. The rotating element inside the vibrators was a cylindrical brass component with a diameter of $20 \mathrm{~mm}$ and a thickness of $8 \mathrm{~mm}$ (weight: $0.022 \mathrm{~g}$ ). The frequency of the rotating element was continuously adjustable between 35 and $40 \mathrm{~Hz}$, which resulted in peak acceleration between 
1.96 and $2.57 \mathrm{~g}$. In this study, four vibrators were applied on the middle part of the quadriceps unilaterally below the electrodes of participants who were lying in the supine position with legs straight. All of them were exposed to a series of 10 sets of LV, each with 1-min duration and 1-min rest between sets, except for $2 \mathrm{~min}$ of rest allowed after the fifth session. This protocol was adapted from the study by Bosco et al. (4), and it has been reported to induce hormonal response following acute WBV exposure. Otherwise, the intermittent muscle contraction with a short rest period between multiple sets was generally expected to produce greater demands on the anaerobic metabolic responses, which may easily lead to an increase in effort and several physiological variables.

\section{BFR device and pressure applied}

In the LV + BFR session, all participants wore a custom-made BFR device around the proximal portion of the unilateral thigh. The device is composed of two inflatable cuffs (made of nylon; width $=9 \mathrm{~cm}$ and length $=70 \mathrm{~cm}$ ), a hand bulb pump with a check valve, a pressure gauge, and rubber tubes, similar to a sphygmomanometer. The two cuffs are connected to the hand bulb by rubber tubes, through which air passes during pumping. Another tube from the bulb connects the pressure gauge, in which the pressure of the cuffs is obtained.

The BFR resistance exercise is often performed using low-intensity resistance exercise combined with BFR. However, the appropriate cuff pressure to be combined with LV for a beginner to perform LV + BFR exercise is not well known. Fahs et al. (11) recommended 160-240 $\mathrm{mmHg}$ as suitable for most individuals for the lower body and indicated typical restrictive cuff pressures for lower body exercises starting the first week of training at $140-160 \mathrm{mmHg}$. Hence, during the pilot work by this study group, the cuff pressure was initially set at $140-160 \mathrm{mmHg}$. After testing the protocol several times, we concluded that participants would not tolerate 10 sets of LV + BFR once the cuff pressure was above approximately $150 \mathrm{mmHg}$. The cuff pressure utilized in this study was set at $140 \mathrm{mmHg}$ to ensure both the safety and protocol completion of all participants. This pressure was consistent with the pressure used in majority of the previous studies on frail individuals (elderly and untrained people) and BFR exercise novices $(1,28)$. To confirm that external BFR combined with LV had an additive effect in the LV + BFR session, the cuff was inflated to the given pressure only when the muscles were being vibration-treated, but not during the rest interval between sets. The intermittent BFR method may be more suitable for untrained populations, because recent studies demonstrated that intermittent BFR resistance exercise causes similar increases in both muscle activation (41) and LA levels (27) as continuous BFR with less discomfort (14) and effort (27).

\section{EMG signal analyses}

Each participant's skin was shaved, abraded, and cleaned with alcohol to minimize impedance, after which bipolar surface electrodes (Ag/AgCL, 3M Health Care, St. Paul, MN) were applied on the belly RF (half the distance from the anterior superior iliac spine to the center of the patella), and the ground electrode was placed over the epicondyle of the tibia. EMG signals were collected by the Noraxon Telemyo DTS EMG system (Noraxon Inc., USA). The EMG signals were amplified $(\times 1000)$ and bandpass-filtered at $10-500 \mathrm{~Hz} \pm 2 \%$ cut-off (Butterworth/Bessels, an anti-aliasing low-pass filter). The electrodes and wireless transducer were secured using tape to minimize disruption during exercise. To evaluate the motor unit requirements, raw EMG signals were processed as the root mean square (RMS) values and were full-wave rectified and low-pass filtered $(12 \mathrm{~Hz})$ over the given periods. Then, the RMS 
values of EMG signals were MVC-normalized according to the isolated MVCs done prior to the session. Subsequently, muscle activity of the RF during LV or LV + BFR was expressed as $\% \mathrm{MVC}$ and then averaged over the 10 sets of each exercise session for data analysis.

\section{Blood sample collection and analyses}

Blood samples were collected from the antecubital vein before and immediately after treatment, as well as 15 - and 30 -min posttreatment at each treatment session. The blood samples were stored at $4{ }^{\circ} \mathrm{C}$ and centrifuged at $1,500 \mathrm{rpm}$ for $30 \mathrm{~min}$ within $2 \mathrm{~h}$ of sampling. Subsequently, the serum samples were stored at $2{ }^{\circ} \mathrm{C}$ for additional serum marker assays. The serum samples were sent to the clinical laboratory for analysis. All the assays were performed according to the manufacturer's instructions. LA was estimated using an automated analyzer Dimension RxL Max Integrated Chemistry System (Siemens Healthcare Diagnostics Inc., Deerfield, IL). Hormones, including the GH, T, and C, were analyzed using a chemiluminometric assay that was performed according to the manufacturers' instructions (GH: Beckman Coulter Inc., USA; T and C: Advia Centaur; Siemens Healthcare Diagnostics, Inc., Tarrytown, NY). The GH level sensitivity and the interassay and intraassay coefficients of variation were $0.002 \mu \mathrm{g} / \mathrm{L}, 2.1 \%-11.3 \%$, and $1.9 \%-14.4 \%$, respectively. The T-level sensitivity and the inter- and intraassay coefficients of variation were $10 \mathrm{ng} / \mathrm{dl}, 2.3 \%-6.2 \%$, and $1.4 \%-4.4 \%$, respectively. The C-level sensitivity and the inter- and intraassay coefficients of variation were $10 \mu \mathrm{g} / \mathrm{dl}, 2.98 \%-$ $3.69 \%$, and $3.99 \%-5.45 \%$, respectively.

\section{Rating of perceived exertion (RPE)}

RPE was measured using the Borg CR-10 Scale immediately at the end of each set of exercise sessions. The participants were asked to rate their perceived exertion by choosing any number on the scale to rate their overall effort. The average value for the 10 sets was calculated for statistical analysis.

\section{Statistical analyses}

Continuous data are expressed as mean \pm standard error. A paired $t$-test was used to compare the EMG amplitude between the LV + BFR and LV sessions. Blood biochemistry values were analyzed using a two-way analysis of variance with a repeated-measures design (trail vs. time). Post hoc test was performed using the least significant difference technique. For the categorical variable (RPE), the Wilcoxon signed-rank test was used, as the data were not measured on a continuous scale. RPE scores are presented as median values and 25 th and 75th percentiles. Statistical significance was set at $p<0.05$ for all tests.

\section{Results}

The paired $t$-test indicated that the EMG\% MVC values of the RF during LV + BFR were significantly higher than those during LV alone $(13.53 \% \pm 4.00 \%$ vs. $8.25 \% \pm 2.20 \%$, $T=2.218, p=0.049$ ).

Regarding LA results, a significant Time $\times$ Trial treatment effect $(F=3.878, p=0.018)$ was found. LA values were significantly increased immediately posttreatment $(p<0.05)$ for the LV + BFR sessions. LA values for LV + BFR were significantly higher $(p<0.05)$ at posttreatment time points than those for LV. In addition, the simple primary effect analysis was significant only for LV + BFR (Fig. 1A). 

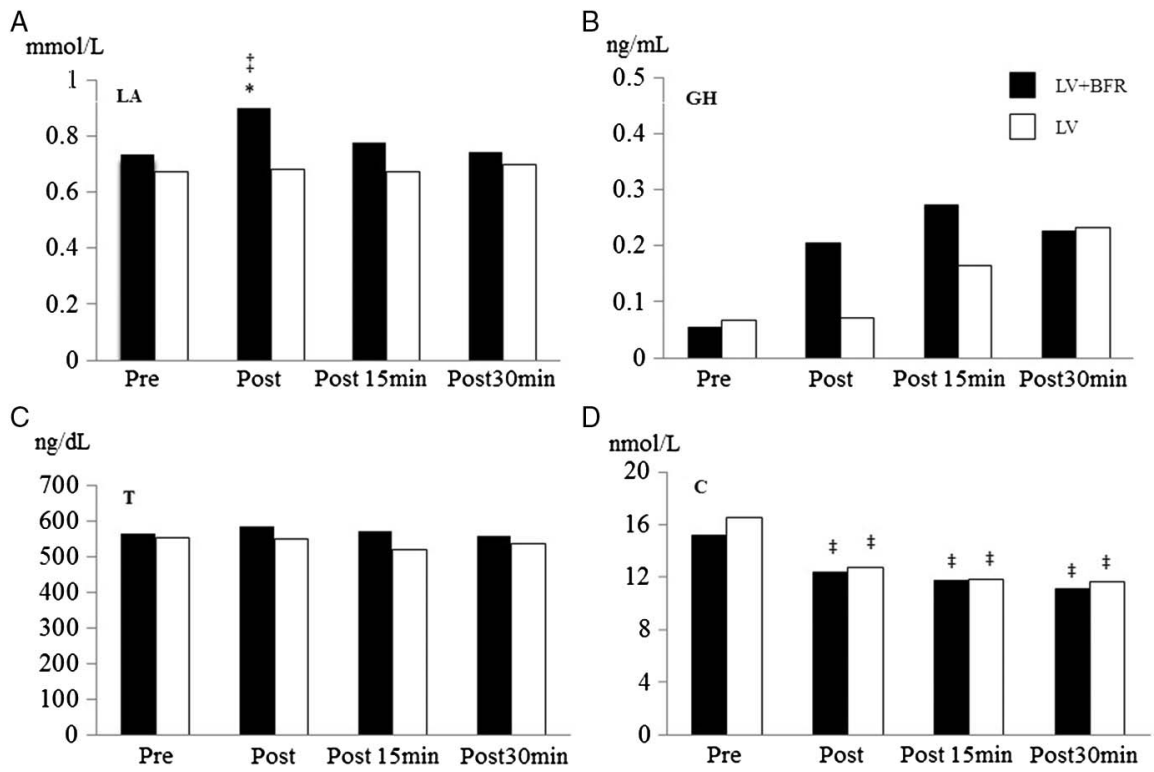

Fig. 1. Comparison of (A) LA, (B) GH, (C) T, and (D) C concentrations (mean \pm standard error) between LV + BFR and LV at various time points. The symbol "*” denotes significant difference between LV + BFR and LV and "t? denotes significant difference compared with the baseline measurements. LA: lactate; GH: growth hormone;

T: testosterone; C: cortisol; LV + BFR: local vibration + blood flow restriction; LV: local vibration

The $\mathrm{GH}$ and $\mathrm{T}$ values are shown in Fig. 1B and $\mathrm{C}$, respectively. Two-way analysis of variance (ANOVA) with repeated-measures design showed no significance for time (GH: $F=0.486, p=0.50 ; \mathrm{T}: F=1.176, p=0.334)$, trial $(\mathrm{GH}: F=2.392, p=0.086$; $\mathrm{T}: F=0.918, p=0.358)$ and Time $\times$ Trial interaction $(\mathrm{GH}: F=0.873, p=0.465 ; \mathrm{T}$ : $1.338 F=0.279$ ) effects.

Figure 1D shows the changes in $\mathrm{C}$ values. Although the two-way ANOVA with repeated-measures design showed that $\mathrm{C}$ values indicated a significant primary effect for time $(F=11.001, p<0.01)$, no evidence was found for Time $\times$ Trial interaction $(F=0.669$, $p=0.577)$ and trail effect $(F=0.616, p=0.449)$. Comparison among time points revealed that compared with the baseline measurements, the $\mathrm{C}$ values were significantly reduced immediately posttreatment $(p<0.05)$ as well as $15(p<0.05)$ and 30 min posttreatment $(p<0.05)$ for both the LV + BFR and LV sessions.

RPE values were obtained by averaging the results of 10 sets of each treatment session. The Wilcoxon signed-rank test revealed that the RPE was higher for the LV + BFR sessions than for the LV sessions [median: 3.65 vs. 2.75; (25th and 75th percentiles: $3.15,4.675$ vs. $1.70,4.02) ; Z=-2.603, p=0.009]$.

\section{Discussion}

This study extends the previous study by applying an external BFR during LV exercise to compare the acute treatment response between LV + BFR and LV sessions. The application of BFR to involuntary muscle contraction has been scarcely explored. The principal findings of this study indicate that the RPE values and working muscle activation in untrained men are 
slightly greater during LV + BFR than during LV session. Increases in LA levels after treatments were detectable only in the LV + BFR session. Neither LV nor LV + BFR elicited changes in GH and T levels. In addition, $\mathrm{LV}$ alone or in combination with BFR decreased C levels.

In this study, the participants were exposed to intermittent LV treatment with a short rest period between multiple sets, which is expected to increase the effort and physiological variables. The specification of the LV device was adapted from Tankisheva et al. (37), which has been reported to increase participants' strength when used regularly. Our data provide the evidence that the RPE values were higher for the LV + BFR session $(3.66 \pm 0.31$, represents moderate to heavy) than for the LV session ( $2.86 \pm 0.39$, represents light to moderate), which supports the notion that external BFR in resistance exercise elicits greater effort during exercise (40). However, the differences in the RPE values between the two sessions are minor.

Muscle contraction through vibration manipulation may produce anaerobic metabolic responses. However, this study failed to find a significant increase in LA levels following LV manipulation. The findings suggested that the magnitude of muscle activity may not be large enough during LV exposure, as the passive muscle contraction of LV showed only a $8.25 \%$ $\pm 2.20 \%$ MVC (EMG RMS) in the RF activity. The increased magnitude of muscle activity during LV exposure is similar to that observed in previous studies, which measured the muscle activity (range: $3.54 \%-11.55 \%$ ) immediately following LV exposure $(3,29)$. Despite this, the RF activity was further increased to $13.53 \% \pm 4.00 \%$ MVC (EMG RMS), and a concomitant increase in LA levels was observed when BFR was applied with LV. A previous study indicated that the increase in LA levels during low-intensity resistance exercise with BFR could partly stimulate muscle activation (40). Altogether, the results suggest that when BFR is applied with LV, the target muscle would be further activated to accommodate the greater metabolic stress as the blood flow is restricted.

In this study, we did not observe any significant changes in the GH and T levels after acute LV manipulation. Our data on GH and T levels disagree with those of Bosco et al. (4), who investigated the effects of acute WBV exercise, but were in general agreement with those of Iodice et al. (18), who used LV as intervention. One possible explanation for the divergent results could be the different vibration modes applied between studies. As only a lesser local muscle were contracted in the LV exercise and this mode of exercise were lack of metabolic demand, which may not elicit the hormonal response like WBV. In addition, LV + BFR also fail to cause significant changes in the GH and T levels. Inagaki et al. (17) indicated that while a single BFR exercise induces GH response, the LA level has to be $>2 \mathrm{mmol} / \mathrm{L}$. Currently, we can only speculate that the BFR when applied with LV did not elicit enough stimulus to induce these hormonal responses. As indicated earlier in this study, LV + BFR induced only slightly greater changes in target muscle activity and LA levels compared with those induced by $\mathrm{LV}$ alone.

Cortisol (C), also known as the stress hormone, is increased through the activation of the hypothalamic-pituitary-adrenocortical axis when an individual encounters stressors (30). The acute increase in $\mathrm{C}$ levels after exercise may reflect the metabolic demands and accelerate the degradation of energy substrates (38). Resistance exercise has often been shown to increase $\mathrm{C}$ levels temporarily $(7,39)$. Contrary to previous findings from resistance exercise, we observed a reduction in $\mathrm{C}$ levels after $\mathrm{LV}$ intervention. The reduction in $\mathrm{C}$ levels after $\mathrm{LV}$ treatment in this study is similar to that reported by a previous study that used either WBV (4) or LV (18) as intervention. Thus, the different results among studies on Clevel responses may be associated with the muscle contraction type (voluntary vs. involuntary induced by TVR). 
Bosco et al. (4) suggested that the reduction in $\mathrm{C}$ levels following acute vibration exposure was attributable to an inhibitory influence on the hypothalamic neurosecretory centers, although the related markers were not detected in their studies. Relative to the increased $\mathrm{C}$ levels as being regarded as under stress, the phenomenon of decrease in $\mathrm{C}$ levels has been linked to relief stress $(9,12,20,21)$. Strategies that relieve a stress response have often been shown to decrease the $\mathrm{C}$ levels concomitantly $(9,12,20,21)$. In addition to the training effect, it is known that the rhythmic muscle contractions evoked by vibration have also been used for the purpose of massage, which has been reported to elicit relaxation, relaxation of myofascial tissues, decrease of emotional tension, and a general sedative effect (19). Buttagat et al. (5) indicated that massage might improve stress-related parameters, including heart rate variability, anxiety, muscle tension, pain intensity, pressure pain threshold, and body flexibility. Furthermore, it has also been reported that an acute massage intervention has decreased $\mathrm{C}$ levels in both blood and saliva $(5,12)$. Altogether, the findings of this study suggest that the LV treatment used in this study may exert a rhythmic massage effect in decreasing the $\mathrm{C}$ levels. In addition, interestingly, a decrease in C levels was also observed in the LV + BFR session in this study. However, the application of BFR with LV did not amplify the response. The BFR resistance exercise mode of $L V+B F R$ used in this study also resulted in differential findings compared with BFR resistance exercise that showed an increase (25) or no change (31) in C levels. Previous research results summarized by Inagaki et al. (17) indicated that an LA level $>7 \mathrm{mmol} / \mathrm{L}$ might be the threshold of metaboreflex to elicit a significant increase in C levels. Since the mechanical stimulation of both LV and LV + BFR on muscle activity is minor, the slightly greater LA levels (Fig. 1A) and muscle activity during LV + BFR compared to those with LV may not be large enough to induce further change in the $\mathrm{C}$ levels.

Regarding the results obtained in this study, some limiting factors become relevant and warrant emphasis. First, it might only be appropriate to extrapolate the findings to populations who seldom engage in regular exercise. Whether physically active people have a similar response to this passive exercise modality ( $\mathrm{LV}+\mathrm{BFR}$ ) compared with untrained people must be clarified in the future. Second, for safety reasons, the cuff pressure applied in this study was $140 \mathrm{mmHg}$, which was consistent with the pressure used in majority of the previous studies on frail individuals (elderly and untrained people) and BFR exercise novices for lower-limb exercise $(1,28)$. This might have led to a disproportionate increase in blood parameter values even when the participants were physically inactive. Third, since the four vibrators were focused on the mid part of the quadriceps, the EMG electrodes attached on the belly RF approaching the vibrators may not fully reflect the maximal muscle activity when exposed to LV, even when the data have been normalized. Finally, a recent study indicated that intermittent BFR resistance exercise causes similar increases in both muscle activation (41) and LA levels (27) as does continuous BFR. Despite this, a review study indicated that continuous rather than intermittent BFR during exercise is preferable to increases in both metabolic demands and motor unit recruitment during low-intensity resistance exercise (11). The potential of $\mathrm{LV}+$ continuous BFR to elicit a greater acute response cannot be entirely ruled out. Therefore, this study may be limited to the intermittent BFR type of modality. Despite these limitations, the findings provide information on target muscle activation and hormonal responses to LV + BFR and LV treatment regimens.

In conclusion, the passive muscle contraction modality of the application of LV + BFR caused a slightly greater effect on the LA response and muscle activity compared with that of LV alone. These minimal effects by BFR as well as LV itself failed to cause changes in the 
GH and T levels. The manipulation of LV and LV + BFR may only have a massage effect as detected by the reduction in the $\mathrm{C}$ levels, probably because the LV elicits an insufficient stimulus to the muscles.

\section{Acknowledgements}

This study was funded by Ministry of Science and Technology (MOST 104-2410-H-110-048), Taiwan. The authors would like to thank all participants for their contribution.

\section{REFERENCES}

1. Abe T, Sakamaki M, Fujita S, Ozaki H, Sugaya M, Sato Y, Nakajima T: Effects of low-intensity walk training with restricted leg blood flow on muscle strength and aerobic capacity in older adults. J. Geriatr. Phys. Ther. 33, 34-40 (2010)

2. Aguayo D, Mueller SM, Boutellier U, Auer M, Jung HH, Flück M, Toigo M: One bout of vibration exercise with vascular occlusion activates satellite cells. Exp. Physiol. 101, 295-307 (2016)

3. Aström C, Lindkvist M, Burström L, Sundelin G, Karlsson JS: Changes in EMG activity in the upper trapezius muscle due to local vibration exposure. J. Electromyogr. Kinesiol. 19, 407-415 (2009)

4. Bosco C, Iacovelli M, Tsarpela O, Cardinale M, Bonifazi M, Tihanyi J, Viru M, De Lorenzo A, Viru A: Hormonal responses to whole-body vibration in men. Eur. J. Appl. Physiol. 81, 449-454 (2000)

5. Buttagat V, Eungpinichpong W, Chatchawan U, Kharmwan S: The immediate effects of traditional Thai massage on heart rate variability and stress-related parameters in patients with back pain associated with myofascial trigger points. J. Bodyw. Mov. Ther. 15, 15-23 (2011)

6. Cardinale M, Bosco C: The use of vibration as an exercise intervention. Exerc. Sport Sci. Rev. 31, 3-7 (2003)

7. Charro MA, Aoki MS, Coutts AJ, Araújo RC, Bacurau RF: Hormonal, metabolic and perceptual responses to different resistance training systems. J. Sports Med. Phys. Fitness 50, 229-234 (2010)

8. Couto BP, Silva HR, Filho AG, da Silveira Neves SR, Ramos MG, Szmuchrowski LA, Barbosa MP: Acute effects of resistance training with local vibration. Int. J. Sports Med. 34, 814-819 (2013)

9. Delarue J, Matzinger O, Binnert C, Schneiter P, Chioléro R, Tappy L: Fish oil prevents the adrenal activation elicited by mental stress in healthy men. Diabetes Metab. 29, 289-295 (2003)

10. Delecluse C, Roelants M, Verschueren S: Strength increase after whole-body vibration compared with resistance training. Med. Sci. Sports Exerc. 35, 1033-1041 (2003)

11. Fahs CA, Loenneke JP, Rossow LM, Thiebaud RS, Bemben MG: Methodological considerations for blood flow restricted resistance exercise. J. Trainol. 1, 14-22 (2012)

12. Field T, Hernandez-Reif M, Diego M, Schanberg S, Kuhn C: Cortisol decreases and serotonin and dopamine increase following massage therapy. Int. J. Neurosci. 115, 1397-1413 (2005)

13. Fahs CA, Loenneke JP, Thiebaud RS, Rossow LM, Kim D, Abe T, Beck TW, Feeback DL, Bemben DA, Bemben MG: Muscular adaptations to fatiguing exercise with and without blood flow restriction. Clin. Physiol. Funct. Imaging 35, 167-176 (2015)

14. Fitschen PJ, Kistler BM, Jeong JH, Chung HR, Wu PT, Walsh MJ, Wilund KR: Perceptual effects and efficacy of intermittent or continuous blood flow restriction resistance training. Clin. Physiol. Funct. Imaging 34, 356-363 (2014)

15. Garber CE, Blissmer B, Deschenes MR, Franklin BA, Lamonte MJ, Lee IM, Nieman DC, Swain DP, American College of Sports Medicine: American College of Sports Medicine position stand. Quantity and quality of exercise for developing and maintaining cardiorespiratory, musculoskeletal, and neuromotor fitness in apparently healthy adults: guidance for prescribing exercise. Med. Sci. Sports Exerc. 43, 1334-1359 (2011)

16. Gauthier AP, Lariviere M, Young N: Psychometric properties of the IPAQ: a validation study in a sample of northern Franco-Ontarians. J. Phys. Act. Health 6, S54-S60 (2009)

17. Inagaki $\mathrm{Y}$, Madarame $\mathrm{H}$, Neya $\mathrm{M}$, Ishii $\mathrm{N}$ : Increase in serum growth hormone induced by electrical stimulation of muscle combined with blood flow restriction. Eur. J. Appl. Physiol. 111, 2715-2721 (2011)

18. Iodice P, Bellomo RG, Gialluca G, Fanò G, Saggini R: Acute and cumulative effects of focused high-frequency vibrations on the endocrine system and muscle strength. Eur. J. Appl. Physiol. 111, 897-904 (2011)

19. Issurin VB: Vibrations and their applications in sport. A review J. Sports Med. Phys. Fitness 45, 324-336 (2005) 
20. Khalfa S, Bella SD, Roy M, Peretz I, Lupien SJ: Effects of relaxing music on salivary cortisol level after psychological stress. Ann. N. Y. Acad. Sci. 999, 374-376 (2003)

21. Lawler SP, Cameron LD: A randomized, controlled trial of massage therapy as a treatment for migraine. Ann. Behav. Med. 32, 50-59 (2006)

22. Loenneke JP, Pujol TJ: The use of occlusion training to produce muscle hypertrophy. Strength Cond. J. 31 , 77-84 (2009)

23. Lowery RP, Joy JM, Loenneke JP, de Souza EO, Machado M, Dudeck JE, Wilson JM: Practical blood flow restriction training increases muscle hypertrophy during a periodized resistance training programme. Clin. Physiol. Funct. Imaging 34, 317-321 (2014)

24. Lu SS, Lau CP, Tung YF, Huang SW, Chen YH, Shih HC, Tsai SC, Lu CC, Wang SW, Chen JJ, Chien EJ, Chien $\mathrm{CH}$, Wang PS: Lactate and the effects of exercise on testosterone secretion: evidence for the involvement of a cAMP-mediated mechanism. Med. Sci. Sports Exerc. 29, 1048-1054 (1997)

25. Madarame H, Sasaki K, Ishii N: Endocrine responses to upper- and lower-limb resistance exercises with blood flow restriction. Acta Physiol. Hung. 97, 192-200 (2010)

26. Martínez-Pardo E, Romero-Arenas S, Martínez-Ruiz E, Rubio-Arias JA, Alcaraz PE: Effect of a whole-body vibration training modifying the training frequency of workouts per week in active adults. J. Strength Cond. Res. 28, 3255-3263 (2014)

27. Neto GR, Novaes JS, Salerno VP, Gonçalves MM, Piazera BK, Rodrigues-Rodrigues T, Cirilo-Sousa MS: Acute effects of resistance exercise with continuous and intermittent blood flow restriction on hemodynamic measurements and perceived exertion. Percept. Mot. Skills 124, 277-292 (2017)

28. Ozaki H, Miyachi M, Nakajima T, Abe T: Effects of 10 weeks walk training with leg blood flow reduction on carotid arterial compliance and muscle size in the elderly adults. Angiology 62, 81-86 (2011)

29. Pamukoff DN, Ryan ED, Blackburn JT: The acute effects of local muscle vibration frequency on peak torque, rate of torque development, and EMG activity. J. Electromyogr. Kinesiol. 24, 888-894 (2014)

30. Papadimitriou A, Priftis KN: Regulation of the hypothalamic-pituitary-adrenal axis. Neuroimmunomodulation 16, 265-271 (2009)

31. Patterson SD, Leggate M, Nimmo MA, Ferguson RA: Circulating hormone and cytokine response to low-load resistance training with blood flow restriction in older men. Eur. J. Appl. Physiol. 113, 713-719 (2013)

32. Pearson SJ, Hussain SR: A review on the mechanisms of blood-flow restriction resistance training-induced muscle hypertrophy. Sports Med. 45, 187-200 (2015)

33. Pope ZK, Willardson JM, Schoenfeld BJ: Exercise and blood flow restriction. J. Strength Cond. Res. 27, 2914-2926 (2013)

34. Shimizu R, Hotta K, Yamamoto S, Matsumoto T, Kamiya K, Kato M, Hamazaki N, Kamekawa D, Akiyama A, Kamada Y, Tanaka S, Masuda T: Low-intensity resistance training with blood flow restriction improves vascular endothelial function and peripheral blood circulation in healthy elderly people. Eur. J. Appl. Physiol. 116, $749-757$ (2016)

35. Suga T, Okita K, Morita N, Yokota T, Hirabayashi K, Horiuchi M, Takada S, Takahashi T, Omokawa M, Kinugawa S, Tsutsui H: Intramuscular metabolism during low-intensity resistance exercise with blood flow restriction. J. Appl. Physiol. 106, 1119-1124 (2009)

36. Tanimoto M, Madarame H, Ishii N: Muscle oxygenation and plasma growth hormone concentration during and after resistance exercise: comparison between "KAATSU" and other types of regimen. Int. J. KAATSU. Training Res. 1, 51-56 (2005)

37. Tankisheva E, Bogaerts A, Boonen S, Delecluse C, Jansen P, Verschueren SM: Effects of a six-month local vibration training on bone density, muscle strength, muscle mass, and physical performance in postmenopausal women. J. Strength Cond. Res. 29, 2613-2622 (2015)

38. Viru M, Litvinova L, Smirnova T, Viru A: Glucocorticoidsin metabolic control during exercise: glycogen metabolism. J. Sports Med. Phys. Fitness 34, 377-382 (1994)

39. Walker S, Taipale RS, Nyman K, Kraemer WJ, Häkkinen K: Neuromuscular and hormonal responses to constant and variable resistance loadings. Med. Sci. Sports Exerc. 43, 26-33 (2011)

40. Yasuda T, Fukumura K, Fukuda T, Iida H, Imuta H, Sato Y, Yamasoba T, Nakajima T: Effects of low-intensity, elastic band resistance exercise combined with blood flow restriction on muscle activation. Scand. J. Med. Sci. Sports 24, 55-61 (2014)

41. Yasuda T, Loenneke JP, Ogasawara R, Abe T: Influence of continuous or intermittent blood flow restriction on muscle activation during low-intensity multiple sets of resistance exercise. Acta Physiol. Hung. 100, 419-426 (2013) 